\title{
Ensayo
}

\section{Encuentros de Francisco de Paula Santander con Alexander von Humboldt y otros científicos en Berlín y París: 1830}

\author{
Alberto Gómez-Gutiérrez
}

Francisco de Paula Santander arribó a París el miércoles 17 de febrero de 1830, un año, tres meses y dos días después de sufrir traslados heteróclitos y estadías improvisadas en medio de su destierro de Colombia, ${ }^{1}$ iniciado en Bogotá el 15 de noviembre de 1828 como consecuencia de su ascendencia y estrecho vínculo con los conspiradores que atentaron contra Simón Bolívar en el Palacio de San Carlos en la noche del jueves 25 de septiembre de 1828:

Salimos [del poblado de (L)ouvres -Hôtel Le Cheval Rouge-]² después de las siete, pagando carísimo la posada y comida, y a las 10 y media llegamos a los arrabales de París, por un camino poblado de alamedas y de pueblos y lleno de carruajes que iban y venían de esta capital. La entrada es por el arrabal San Martín, el cual pasado, se llega a una puerta donde se registran los carruajes; a mí me preguntó uno si tenía que declarar algo y, respondido negativamente, pasamos inmediatamente. Tomamos el boulevard de los italianos y atravesamos por junto a la bolsa de los mercaderes y llegamos al Hôtel Boston, calle Vivienne, ${ }^{3}$ el cual había salido en suerte de cinco que tiramos para saber a dónde nos alojaríamos. Me dieron un salón decente y dos cámaras por diez francos diarios, y los tomé sólo por una semana. La leña es cara y la comida a la carta no es barata. Yo hice buscar un alojamiento más barato y nada se realizó.

Al día siguiente, Santander se vio con "el señor Pío Rengifo, colombiano", ${ }_{4}^{4}$ a quien había conocido en Bogotá:

$[\mathrm{M}]$ e hizo muchos ofrecimientos y entre ellos dinero. Se encargó de buscarme un alojamiento. Salí a dar un paseo y lo hice por el famoso edificio llamado Palais Royal, que merece ser llamado famoso. Allí se encuentran tiendas de ropa, cafés, restaurantes, joyerías, etc. El patio está cubierto de alamedas y a un lado hay una fuente que tiene varios chorros de agua bastante altos. En este palacio vive el duque de Orleans [...]. En los diarios he leído que continúa la insurrección de Venezuela y que se ha ofrecido al presidente de los Estados Unidos de América la medalla emitida para perpetuar la memoria de la salvación de Bolívar, la noche del 25 de septiembre de 1828 .

El viernes 19 lo visitó Joaquín Acosta Pérez, quien se había enterado por su amigo Pío Rengifo y Díez de la Fuente (1799-1867) que el general Francisco de Paula Santander había llegado el miércoles 17 al Hôtel Boston de la rue Vivienne. Acosta acudió con José Domingo, ${ }^{5}$ su hermano mayor, y Vicente Concha Maldonado (1809-1830), a quien Santander se refirió como su pariente. ${ }^{6}$ Dice así Santander en su Diario de viaje:

Febrero 19. Viernes: Joaquín Acosta, oficial colombiano, me ha visitado esta mañana. Vive en la rue de l'Ouest N. ${ }^{\circ}$ 9. Estuvieron Domingo Acosta y Vicente Concha, mi pariente. ${ }^{7}$

Joaquín Acosta había establecido previamente un estrecho vínculo en París con Alexander von Humboldt, y este había surgido años atrás en medio del viaje americano de Humboldt y Bonpland, cuando estos viajeros pasaron por Guaduas entre Honda y Bogotá, y se alojaron en casa de los Acosta. Humboldt mismo se refirió a este antecedente en una nota escrita a Joaquín Acosta en París a finales del mes de marzo de 1827:

Tendré el más vivo placer en recibir al señor Roche y á Domingo Acosta, cuya respetable familia (hace siglos!) tuvo para mí tantas bondades. Venga á verme el sábado á las 8 y media, si esto no lo molesta demasiado. Su adicto, Humboldt. ${ }^{8}$ 
El Diario de viaje de Francisco de Paula Santander resulta particularmente útil para precisar quiénes habrían integrado en esos años el círculo de exiliados y viajeros al que Humboldt había llamado ya "inos colombiens!" en una nota a Joaquín Acosta en el mes de julio de 1826. ${ }^{9}$ Además de los Acosta, Rengifo y Concha, Santander refiere, en orden cronológico, sus encuentros con José María Salazar; ${ }^{10}$ Jerónimo Torres; ${ }^{11}$ Delgado $;{ }^{12}$ el general Herrán $;{ }^{13}$ Roche, hijo de Pepa Domínguez; ${ }^{14}$ el cuñado de Hurtado ${ }^{15}$ Santamaría, que estuvo de ministro en Méjico ${ }^{16}$ Santiago Rodríguez, diputado en la convención de Ocaña ${ }^{17}$ Palacios ${ }^{18}$ y Ezequiel Rojas. ${ }^{19}$ Pero con quien más tiempo pasa Santander, además de Pacho ${ }^{20}$ y Juan y Pepe, ${ }^{21}$ sus compañeros de viaje, es con Joaquín Acosta, con quien visita museos, academias y diversos personajes de la ciencia y de la política.

Una de estas visitas resulta muy significativa en el dominio de la ciencia. En efecto, el lunes $1^{\circ}$ de marzo, Santander registró en su Diario de viaje una visita excepcional:

$1^{\circ}$ - Lunes: Anoche estuve en la diversión de títeres y sombras chinescas, en el Palacio Real, por no tener qué hacer. Vino Acosta (Joaquín) para ir a casa del general Lafayette, lo encontramos y me hizo una acogida muy amable. Hablamos de las cosas de Colombia y de Bolívar. Quedé de concurrir a las soirées de los martes. De allí fuimos a casa del señor Jullien, luego entramos al magnífico jardín del Luxemburgo mucho más grande que el de las Tullerías y pasamos al Instituto, donde presencié una sesión de la Academia de Ciencias, presidida por Girard físico. En ella vi a los siguientes miembros: La Croix, autor de una obra muy conocida de matemáticas (es cojo), a Fourier, matemático y secretario de la academia, Cuvier, también secretario, el primer naturalista de Europa, Wardem autor de la estadística de los Estados Unidos, Thénard, y Chapsal ex-ministro del interior del tiempo de Napoleón, ambos químicos afamados; Arago, astrónomo director del Observatorio, Freycinet, marino que ha dado vuelta al mundo; Puissant, coronel geógrafo, Virey naturalista,. Desfontaines botánico, Mathieu, astrónomo, Moreau de Jonnes, estadístico, Brongniart, naturalista director de la fábrica de porcelana de Sevres, Biot, físico, el barón Portal, anatómico y Legendre, geómetra. Yo fui presentado a Warden, a Arago, a Puissant, a Brongniart; Wardem me llevó a la biblioteca donde está la estatua de Voltaire desnudo y sentado y los bustos de Franklin, Malesherbes, La Grange, Berthollet, Volney [...].

El señor Arago quiso hacerme sentar en las sillas destinadas a los extranjeros ilustres que visitan la Academia; me negué a ello. La sala de la Academia es grande: los académicos se sientan formando elipse y en medio de ella: cada uno tiene tintero y papel por delante. La sesión se abrió dando cuenta de la correspondencia dirigida a la Academia y luego leyó una memoria un miembro, para sostener el sistema nuevo que había presentado, al cual se había referido Cuvier. ${ }^{22}$

Dos semanas después, el sábado 13 de marzo, Santander salió a visitar la École de Commerce de Paris con Marc-Antoine Jullien (1775-1848), escritor revolucionario y periodista parisino, fundador y redactor principal de la Revue encyclopédique a partir de 1819. Pero antes de pasar a la margen derecha del río Sena que divide en dos la ciudad, decidieron visitar "el Jardín del Rey o Jardín Botánico", en donde les presentaron al "naturalista [Geoffroy] de Saint-Hilaire, miembro de la Academia Real de Ciencias, el cual habló de la memoria que trabajaba contra la opinión de M. [Georges] Cuvier sobre la analogía de la organización [anatómica] animal”. ${ }^{23}$

El miércoles 7 de abril, después de haber visitado en los primeros 15 días de marzo el Institut de France y luego lo que hoy se conoce como el Musée National d'Histoire Naturelle, altos referentes de la ciencia francesa, Santander hizo una tercera visita de importancia en ese mismo dominio:

Fui con J[oaquín] Acosta al Observatorio a visitar al señor Arago que tuvo la bondad de mostrarnos el establecimiento, hay en él pocos instrumentos, pero los que hay son magníficos. Una luneta ecuatorial que sigue el movimiento de las estrellas, un gran círculo, diferentes telescopios, agujas de la última perfección y el famoso anteojo que sirve para determinar la meridiana. Hay modo de conocer la electricidad de la atmósfera por medio de un hilo de plata que está prendido de un pararrayo y termómetros enterrados para conocer el grado de calor de la tierra a 25 pies de profundidad. El señor Arago nos regaló un anuario de longitudes. (En el Observatorio está la sala llamada bureau des longitudes) [...]. En otra sala del Observatorio están los bustos de Laplace, La Grange, D’Alembert, Bréguet, La Condamine, Newton y la estatua de Dominique Cassini. ${ }^{24}$ 
Acosta, quien se había conectado con la intelligentsia científica de París gracias a su amistad con Humboldt, salió de viaje en el primer semestre de 1830 en hacia Londres con Pío Rengifo. El general Santander, por su parte, partió a comienzos del mes de junio con destino a Inglaterra, tras repetidas noticias -sucesivamente desvirtuadas- de la muerte de Bolívar, quien por esos días iba ya en camino hacia su último suspiro en la quinta de San Pedro Alejandrino en las afueras de Santa Marta el 17 de diciembre de ese mismo año. Este suceso abriría, antes de lo previsto, el espacio político para el retorno de Santander y para su posesión en la presidencia de Colombia a partir de 1832.

Antes de regresar a Colombia, Santander pasó nuevamente por París con varias escalas previas incluyendo la capital alemana, en donde se encontró el martes 17 de agosto con Humboldt. Este fue el primer encuentro de Santander con el prusiano, y se dio por casualidad en medio de su visita a uno de los museos de esa ciudad. Al día siguiente, miércoles 18 de agosto, Humboldt visitó a Santander y el general le devolvió la visita a Humboldt el jueves 19 de esa misma semana. El sábado 21 Humboldt lo invitó nuevamente para almorzar juntos el domingo:

¿Me haría el honor, Sr. General, de venir a mi casa a almorzar mañana domingo (a las nueve de la mañana) [...]. Mil tributos de admiración y respeto. Alexander Humboldt. ${ }^{25}$

Santander reporta en su Diario de viaje que el encuentro tuvo efectivamente lugar, y que además salieron a pasear por los jardines del rey:

Agosto 22. Domingo: Almorcé con Humboldt y había allí 8 personas más, todas las cuales eran literatos con excepción de un caballero florentino que pertenece a la corte de Toscana y viaja en Alemania. Fuimos al parque de Charlottenburg, residencia del rey en la primavera, a media milla de la ciudad. ${ }^{26}$

El sábado 28 de agosto Santander anota: "Me despedí por escrito del barón de Humboldt, por estar en el campo con el rey"27. Pero esta no sería la última vez que habría un contacto entre el barón prusiano y el futuro presidente de la Nueva Granada antes de su retorno a la patria. Se conserva evidencia impresa de una nota manuscrita que Humboldt envió a Santander en París, en la siguiente primavera, el martes 19 de abril de 1831:

No es sino por haber refundido durante varios días la amable nota de S[u] E[xcelencia] el Señor General Santander que el Señor de Humboldt tardó en ofrecerle su afectuoso homenaje, proponiéndole de honrarle con su visita el Jueves a las cinco horas. El Sr de H[umboldt] no anotó la dirección del Señor General. ${ }^{28}$

El registro de este encuentro en el Diario de viaje de Santander, dice así:

Jueves. He visitado a Humboldt, y aprueba mi resolución de permanecer en Europa todavía. He escrito sobre esto un artículo a los diarios de París. Con fecha 15 he dirigido una representación al congreso de Colombia pidiendo se publique mi causa y mi representación a Bolívar enviada de Bocachica. ${ }^{29}$
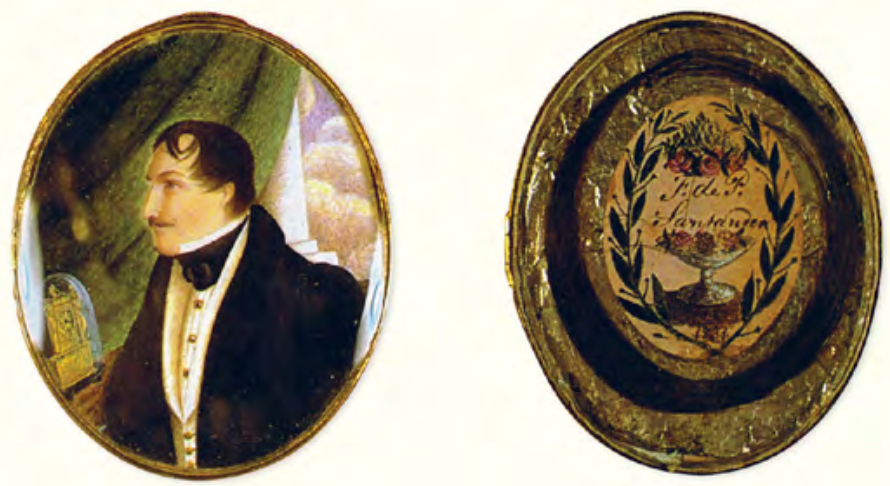

Francisco Evangelista González. "F. de P. Santander”, miniatura (c. 1831). Tomado de Moreno de Ángel, Pilar y Horacio Rodríguez Plata. Santander. Su iconografía. Bogotá: Litografía Arco, 1984, p. 82. 
En conclusión, con base en esta relación de notas y encuentros científicos y no científicos de Francisco de Paula Santander en París y en Berlín, que podrían ser fuente de sucesivos trabajos históricos, se puede sustentar, por ejemplo, que Alexander von Humboldt, después de haber recorrido la Nueva Granada entre 1800 y 1803 interactuando con decenas de neogranadinos en aquellos días, no solo siguió aconsejando a los nuevos científicos en ciernes -como Joaquín Acosta-, sino que se había convertido también en esos días en oráculo político de quienes ahora se llamaban colombianos y, en particular, de su máxima autoridad en el exilio.

\section{Notas al final}

1 La secuencia de traslados y estadías registrada con los subtítulos correspondientes por Santander, inicia con Diario de navegación de Puerto Cabello a Hamburgo, luego su Llegada a Hamburgo (15 de octubre de 1829) y Salida de Hamburgo (14 de diciembre de 1829), continuando con las siguientes localidades: Hannover - Gottinga - Cassel - Marburg - Francfort del Mein Darmstad - Maguncia - Coblenza - Bonn - Colonia - Aquisgran - Francia-París - París (febrero 17) - Salida de París en viaje a Inglaterra (4 de junio de 1830) - Inglaterra - Londres - Brujas - Breda-Rotterdam - La Haya-Leyden-Haarlem-Amsterdam - Apreciaciones sobre Holanda y los holandeses - Llegada a Hamburgo el 27 de julio de 1830 - Salida de Hamburgo el 14 de agosto - Prusia-Berlin-Berlín-Apreciaciones sobre Prusia-Sajonia-Dresde - Dresde - Baviera - Nuremberg - Ratisbona - Munich - Tirol Alemán - Innsbruck - Tirol Italiano Trento - Italia - Reino Lombardo - Véneto - Verona - Mantua - Venecia - Padua - Estados Pontificios - Ferrara - Bolonia - Toscana - Florencia - Roma y Estados Pontificios - Roma Pisa - Liorna - Lucca - Piamonte o Reino de Cerdeña - Génova - Lombardía-Pavía-MilánSuiza - Lausana-Ginebra-Francia-Dijon-Paris-Inglaterra-Londres - York-Edimburgo - Glasgow - Irlanda - Dublin - Banor - Liverpool - Manchester - Birmingham - Oxford Londres - París. En el año 1831, registró su paso a América, así: Estados Unidos - Nueva York - Filadelfia - Baltimore - Washington - Observaciones sobre los Estados Unidos - Nueva York - Pensilvania, en donde el diario termina abruptamente. Para acceder a una edición digital del Diario del general Francisco de Paula Santander en Europa y los EE. UU. 1829-1832, transcrita y anotada para el Banco de la República en 1963 por Rafael Martínez Briceño, véase: http:// babel.banrepcultural.org/cdm/ref/collection/p17054coll18/id/458. El presente texto tomará como referencia bibliográfica para las citas incluidas a partir del Diario, la edición de Mario Germán Romero titulada Santander en Europa. Diario de viaje 1829-1832. Bogotá: Fundación Francisco de Paula Santander, 1989. Edición digital: http://www.bdigital.unal.edu.co/7802/1/Santander_en_ Europa_1829-1832.html\#4c

2 Esta población está registrada en la transcripción del Diario de viaje de Francisco de Paula Santander como "Douvres", pero se refiere evidentemente a la población de Louvres, a mitad de camino entre Gournay - pueblo en donde pernoctó el día anterior-, y París. Véase Santander en Europa. Diario de viaje 1829-1832, 1989, 1, p. 99.

3 El Hôtel Boston estaba situado en el número 13 de la rue Vivienne. Simón Bolívar se había alojado en el número 3 de esa misma calle (Hôtel des Étrangers) en 1804 cuando, muy joven, había viajado a París tras la muerte de su esposa.

4 Pío Rengifo y Díez de la Fuente (1799-1867), político y comerciante nacido en Cali, padre de Pío Rengifo Martínez, doctorado en medicina en la Universidad de París en 1863, y especializado en Alemania e Inglaterra. Una vez establecido en Bogotá, en 1873, se vinculó a la recién fundada Sociedad de Medicina y Ciencias Naturales que llegaría a convertirse en 1891 en la Academia Nacional de Medicina. Rengifo Martínez fue catedrático en la Universidad Nacional y redactor fundador de la Revista Médica de Bogotá. En 1880 viajó a los Estados Unidos y se radicó en Nueva York, donde ejerció su profesión. Ibidem, p. 100.

5 Joaquín y José Domingo Acosta Pérez eran hijos de José de Acosta Gaine (1745-1803), anfitrión de Humboldt y Aimé Bonpland a su paso por Guaduas en 1801. José de Acosta, inmigrante español, agricultor y comerciante nombrado administrador de la Real Renta de Tabacos y demás rentas estancadas, además de corregidor de Guaduas por el virrey Ezpeleta en 1789, había casado en segundas nupcias con María Soledad Pérez Marchán, con quien tuvo ocho hijos, entre los cuales se destacaron dos: José Domingo Acosta Pérez (1792-1847), fundador de la primera biblioteca pública de Guaduas, secretario de la primera misión diplomática de la Nueva Granada en la Gran Bretaña, y el general Joaquín Acosta Pérez (1799-1852), prócer de la Independencia, geógrafo, hombre de ciencia, historiador, estadista y generoso benefactor, quien se casó con la norteamericana Caroline Kemble Rou, y fueron padres de Soledad Acosta Kemble (1833-1913), 
reconocida literata, historiadora y poeta de la segunda mitad del siglo XIX, casada con José María Samper Agudelo (1828-1888). Joaquín Acosta fue el principal discípulo colombiano de Humboldt en París. Para pormenores de los vínculos de Humboldt y los colombianos en los años 20 del siglo XIX en París, particularmente con Joaquín Acosta, véase Gómez Gutiérrez, Alberto. Humboldtiana neogranadina. Bogotá: Cesa / Pontificia Universidad Javeriana / Universidad de los Andes / Universidad del Rosario / Universidad Eafit / Universidad Externado de Colombia, 2018, I, 2, pp. 260-331.

6 La referencia a Vicente Concha "su pariente" es valiosa desde el punto de vista histórico, puesto que no solo se trataba del hijo del coronel patriota Mariano José Concha Sánchez (1785-1830) y de Bárbara Maldonado Omaña, hija, a su vez, de Teresa Omaña Santander (por tanto, Concha era sobrino doble del general), sino que comprueba que este personaje, desterrado a Jamaica en 1829 en calidad de adversario de Bolívar, pasaría por París antes de volver a su patria para morir junto a su padre en la batalla de Cúcuta, en noviembre de 1830, al enfrentarse a las tropas del presidente Rafael Urdaneta (1788-1845). De hecho, Santander refiere en su Diario de viaje que, en abril 10 había "escrito al coronel Concha con su hijo Vicente", y luego, en abril 16, "Hoy he despachado a mi negro [Juan] Cruz Cabrejo para Puerto Cabello, con Vicente Concha, y al efecto le he escrito al arzobispo de Caracas para que lo remita para Bogotá. Al negrito le he entregado su carta de libertad" (Santander en Europa. Diario de viaje 1829-1832, 1989, 1, 138 y 140).

7 Santander en Europa. Diario de viaje 1829-1832, 1989, 1, p. 101.

8 Humboldt, Alexander, citado en Acosta de Samper, Soledad. Biografía del General Joaquín Acosta. Bogotá: Librería Colombiana Camacho Roldán \& Tamayo, 1901, p. 110.

9 La nota de Humboldt decía así: "Je serais heureux de pouvoir vous offrir des billets et je serais bien aise si M. Acosta vouloit avoir l'extrème bonté de passer chez moi demain samedi à trois heures pour le consulter sur Cajicá. Donnez, de grace, un billet, en mon nom, à M. Restrepo ou à un de les amis jnos colombiens! Mille amitiés [Estaría encantado de ofrecerle billetes y me gustaría mucho que el Sr. Acosta tuviera la amabilidad de pasar por mi casa mañana sábado a las tres en punto para consultarle sobre Cajicá. ¡Por favor, dele un boleto a mi nombre al Sr. Restrepo o a uno de los amigos ¡nuestros colombianos! Mil amistades] Humboldt” Para una relación más amplia de los primeros colombianos en París, sus funciones, fechas de estadía y los lugares en donde se alojaron en los años veinte del siglo XIX, véase Gutiérrez Ardila, Daniel. "Los primeros colombianos en París (1824-1830)". Anuario Colombiano de Historia Social y de la Cultura, 2009, 36 (1): 89-124.

10 José María Salazar Arango (1784-1830), diplomático y representante de Colombia en Europa.

11 Jerónimo Torres Tenorio (1771-1838), uno de los más prestigiosos abogados y académicos de la época. Fue un progresista ciudadano que participó en la Independencia y llegó a ser consejero de Estado, director de la Casa de Moneda en Santafé, encargado de negocios en Francia y contador general de Hacienda. Uno de sus hermanos, Camilo Torres Tenorio (1766-1816) hizo estudios de derecho en el Colegio Mayor de Nuestra Señora del Rosario de Bogotá, del cual llegó a ser Vicerrector en 1792. Autor del "Memorial de Agravios", miembro de la Junta Suprema de Bogotá y presidente del Congreso de las Provincias Unidas en el proceso de la Independencia neogranadina colombiana. En el año 1816 buscó exiliarse con otros próceres derrotados por el ejército español. Pero fue capturado y fusilado el 5 de octubre de ese mismo año.

12 Dos Delgados contemporáneos de Santander y posibles viajeros a París fueron: a) el abogado republicano Juan Antonio Delgado Martínez (1794-c. 1865), perseguido por los realistas en 1813 y, posteriormente, jefe político del cantón de Popayán y luego de Cali; b) su sobrino Julián Antonio Delgado Martínez (n. 1797), tesorero del Cauca entre 1821 y 1827.

13 Pedro Alcántara Herrán Zaldúa (1800-1872), último ministro de guerra de la Gran Colombia y secretario de la legación ante la Santa Sede de 1830 a 1832. Herrán fue presidente de la República de Colombia entre 1841 y 1845.

14 Vicente Roche Domínguez (n. 1800), quien participó en la constitución de la primera república en calidad de subteniente de artillería en Santafé en 1824, era hijo del español José Manuel de la Roche O’Ryan (1773-c. 1820) y María Josefa Domínguez de Castillo (1782-c. 1850).

15 Puede referirse a un cuñado de Manuel José Hurtado Arboleda (1782-1845), prócer de la Independencia, senador de la Gran Colombia y ministro plenipotenciario en Inglaterra, quien se había casado con la panameña Carmen Díaz.

16 Miguel Santa María (1789-1837), escritor y político liberal y republicano nacido en Veracruz, México. Santa María llegó a ser ministro plenipotenciario de la Gran Colombia en su país natal entre 1822 y 1828, a pesar de haber sido desterrado por el emperador Agustín Cosme Damián de Iturbide y Aramburu (1783-1824), quien renunció a la corona en 1823 cuando todavía Santa María se encontraba en el puerto de su patria chica. 
17 Santiago Rodríguez, uno de los 23 venezolanos que participó en la Convención de Ocaña.

18 General José Leandro Palacios de la Plaza (1782-1836), caraqueño, ministro plenipotenciario de Colombia en Brasil hasta 1828; pasó a Francia, donde no fue reconocido por Carlos X. El general Santander, en su extensa carta a su íntimo amigo y paisano Francisco Soto Montes de Oca (17891845), iniciada en Roma el 12 de diciembre de 1830, y ya con fecha del 29 de marzo de 1831 desde Ginebra (más de tres meses después de la muerte de Bolívar) le pide que "si hay ya libertad de imprenta, haga publicar que Lafayette se empeñó en que me reconciliase con Bolívar, que yo en presencia de los señores Miguel Santa María y Leandro Palacio, le respondí que nuestras diferencias nacían de la oposición de nuestros principios políticos y no de personalidades, y que era preciso que uno de los dos abandonase los suyos; que yo no podía renunciar a los míos porque eran los de la libertad por lo cual había hecho Colombia grandes sacrificios, y ni mi honor ni mi conciencia me permitían traficar con ellos" (Santander en Europa. Cartas de viaje 1829-1832. 1989, 3, p. 101).

19 Ezequiel Rojas Ramírez (1803-1873), abogado y político santanderista implicado en la conspiración septembrina contra Bolívar, fue diplomático y representante de Colombia en Europa y, más adelante, propuesto como primer rector de la Universidad Nacional en 1867. En razón de quebrantos de salud, fue reemplazado por Manuel Ancízar Basterra (1811-1882), quien ejerció el cargo entre 1868 y 1871.

20 Francisco de Paula Evangelista González (n. 1807), quien había embarcado con Santander y con Ezequiel Rojas. Su amigo 'Pacho', como le decía Santander, dejó, al menos, dos retratos en miniatura fechados en 1831.

21 Juan Cruz Cabrejo y José Delfín Caballero, criados de Santander. El general hizo referencia al segundo en una de sus epístolas, en la que menciona que "Pepe, gordo y grande, trabaja como ebanista muy regularmente" (Santander en Europa. Cartas de viaje 1829-1832, 1989, 3, p. 113). También, al regresar a su patria vía Nueva York, escribió a su hermana: “Juan está loco por llegar a Bogotá. Pepe trabaja con provecho. Si los vieras cómo están de petimetres y decentes te habría de dar gusto. Yo los tengo así porque me sirven y porque es honor mío en países extranjeros" (Santander en Europa. Cartas de viaje 1829-1832, 1989, 3, p. 166).

22 Santander en Europa. Diario de viaje 1829-1832, 1989, 1, pp. 107-108.

23 Ibidem, 1, p. 115-116. La polémica entre Saint-Hilaire y Cuvier en 1830 tuvo resonancia internacional. En términos de una enciclopedia contemporánea: "El célebre debate entre Geoffroy y Cuvier se desarrolló oficialmente en forma de comunicaciones y discusiones públicas en la Academia de las Ciencias hasta abril de 1830, pero también, de modo no oficial, en revistas e incluso en la prensa diaria. La polémica, que ilustra el apogeo de la anatomía comparada como disciplina central en el conjunto de las ciencias naturales, se concluyó abruptamente con la inesperada muerte de Cuvier en 1832". Véase https://es.wikipedia.org/wiki/\%C3\%89tienne_ Geoffroy_Saint-Hilaire

24 Santander en Europa. Diario de viaje 1829-1832, 1989, 1, p. 109.

25 Santander en Europa. Cartas de viaje 1829-1832, 1989, 3, p. 320. Debe atenderse al hecho de que el barón firmaba a veces sin las partículas "de" o "von", en especial cuando se dirigía a un ciudadano patriota como Santander.

26 Santander en Europa. Diario de viaje 1829-1832, 1989, 1, p. 217.

27 Ibidem, 1, p. 222.

28 Ibidem, 1, p. 215.

29 Ibidem, 2, p. 103. 